\title{
Stability properties of Plethysm: new approach with combinatorial proofs (Extended abstract)
}

\author{
Laura Colmenarejo $\|^{p}$ \\ ${ }^{1}$ Departamento de Álgebra, Universidad de Sevilla, Sevilla, Spain
}

\begin{abstract}
Plethysm coefficients are important structural constants in the theory of symmetric functions and in the representations theory of symmetric groups and general linear groups. In 1950, Foulkes observed stability properties: some sequences of plethysm coefficients are eventually constants. Such stability properties were proven by Brion with geometric techniques and by Thibon and Carré by means of vertex operators. In this paper we present a new approach to prove such stability properties. This new proofs are purely combinatorial and follow the same scheme. We decompose plethysm coefficients in terms of other plethysm coefficients (related to the complete homogeneous basis of symmetric functions). We show that these other plethysm coefficients count integer points in polytopes and we prove stability for them by exhibiting bijections between the corresponding sets of integer points of each polytope.

Résumé. Les coefficients du pléthysme sont des constantes de structure importantes de la théorie des fonctions symétriques, ainsi que de la théorie de la représentation des groupes symétriques et des groupes généraux linéaires. En 1950, Foulkes a observé pour ces coefficientes de phénomènes de stabilité: certaines suites de coefficients du pléthysme sont stationnaires. De telles propriétés ont été démontrées par Brion, au moyen de techniques géométriques, et par Thibon et Carré, au moyen d'opérateurs vertex. Dans ce travail, nous présentons une nouvelle approche, purement combinatoire, pour démontrer des propriétéséde stabilité de ce type. Nous décomposons les coefficients du pléthysme comme somme alternées de coefficients de pléthysme d'un autre type (liés à la base des fonctions symétriques sommes complètes), qui comptent les points entiers dans des polytopes. Nous démontrons la stabilité des suitres de ces coefficients en exhibant des bijections entres les ensembles de points entiers des polytopes correspondants.
\end{abstract}

Keywords: Combinatorial representation theory, symmetric functions, plethysm, combinatorial intepretation of coefficients, stability

\section{Introduction}

Understanding structural constants is one of the most important problems in representation theory, and even in simplest cases we can find unsolved problems.

\footnotetext{
†Email: laurach@us.es. Supported by project MTM2010-19336 and FEDER, and Junta de Andalucía under grants FQM333 and P12-FQM-2696

1365-8050 @ 2015 Discrete Mathematics and Theoretical Computer Science (DMTCS), Nancy, France
} 
Recall that any (finite-dimensional, complex, analytic) linear representation $V$ of $G L_{n}(\mathbb{C})$ decomposes as a direct sum of irreducible representations:

$$
V \approx \bigoplus m_{\lambda} S_{\lambda}\left(\mathbb{C}^{n}\right)
$$

where the $m_{\lambda}$ are non-negative integers called multiplicities and the $S_{\lambda}\left(\mathbb{C}^{n}\right)$ are the irreducible representations of $G l_{n}(\mathbb{C})$, which are indexed by partitions of length at most $n$.

Important families of structural constants appear in non-trivial constructions of new representations from old ones. Three of them are particularly importants in a more combinatorial context. Firstly, consider the tensor product of two irreducible representations $S_{\mu}\left(\mathbb{C}^{n}\right) \otimes S_{\nu}\left(\mathbb{C}^{n}\right)$ and decompose it into irreducibles. Then, the multiplicities arising from this are the Littlewood-Richardson coefficients, $c_{\mu \nu}^{\lambda}$. Combinatoric interpretations behind this family are well understood: for example, they count Littlewood-Richardson Young tableaux or integral points in the hive polytopes. Moreover, these interpretations are efficient tools for proofs and computations.

Next, consider an irreducible representation of $G L_{m n}(\mathbb{C})$ as a representation of $G L_{m}(\mathbb{C}) \times G L_{n}(\mathbb{C})$ by means of the Kronecker product of matrices and decompose the resulting representation into irreducibles. Then, the multiplicities arising from this operation are the Kronecker coefficients, $g_{\lambda \mu}^{\nu}$. In this case, combinatorial interpretations are known, but just in particular cases. For example, C. Ballantine and R. Orellana [BO07] have an interpretation in terms of Kronecker tableaux of the Kronecker coefficients when one of the partitions has just two parts.

Finally, the plethysm coefficients $a_{\lambda, \mu}^{\nu}$ are the multiplicities obtained when we apply a Schur functor $S_{\lambda}$ to an irreducible representation $S_{\mu}\left(\mathbb{C}^{n}\right)$ and we decompose the resulting representation into irreducibles.

One of the major problems in combinatorial representation theory is to find similar general interpretations for the Kronecker coefficients and the plethysm coefficients, akin to those known for the LittlewoodRichardson coefficients. For the Kronecker case, Murnaghan [Mur38] and Littlewood [Lit56] observed that some sequences stabilize (they are eventually constant). We can find results about these stability phenomena in papers of E. Vallejo [Val99], L. Manivel [Man14], I. Pak and G. Panova [PP14] or E. Briand, R. Orellana and M. Rosas [BOR11], for example.

The plethysm coefficients also show stability properties. Some of them was observed by Foulkes [Fou50] and in 1990's they were proved by Carré and Thibon [CT92], using vertex operators and other combinatorial arguments in symmetric functions, and Brion [Bri93] for algebraic groups in general (rather than just general linear groups) and using tools from geometric representation theory.

In this paper, we consider some of them.

Theorem 1.1 The sequences of general terms

$$
\left\{a_{\lambda+(n), \mu}^{\nu+n \cdot \mu}\right\},\left\{a_{\lambda+(n), \mu}^{\nu+(n \cdot|\mu|)}\right\},\left\{a_{\lambda, \mu+(n)}^{\nu+(n \cdot|\lambda|)}\right\}
$$

stabilize.

In the following, we will write $\lambda(n), \mu(n)$ and $\nu(n)$ to mean the different partitions considering in Theorem 1.1

We give a new combinatorial proof, that we sketch now. The stability of the sequences of plethysm coefficients $a_{\lambda(n), \mu(n)}^{\nu(n)}$ that we consider will follow from the stability of the corresponding sequences of plethysm coefficients $b_{\lambda(n)+\omega, \mu(n)}^{\nu(n)+\omega^{\prime}}$. These plethysm coefficients, that we call $h$-plethysm coefficients, are 
the coefficients of the monomial function $m_{\nu}$ in the plethysm $h_{\lambda}\left[s_{\mu}\right]$, where the $h_{\lambda}$ are the complete homogeneous functions and the $s_{\mu}$ are the Schur functions. We show that the coefficients $b_{\lambda, \mu}^{\nu}$ have a combinatorial interpretation in general: they count integer points in a polytope, $Q(\lambda, \mu, \nu)$. Then, in order to prove the stability for them, we built an injection from the polytope corresponding to $b_{\lambda(n), \mu(n)}^{\nu(n)}$ to the polytope corresponding to $b_{\lambda(n+1), \mu(n+1)}^{\nu(n+1)}$. Finally, we show that these injections are surjective for $n$ big enough.

The idea of testing the stability of some coefficients through other coefficients can be also found in other references, as in the paper of Stembridge [Ste14], where he uses a similar idea for proving stability in case of Kostka coefficients and Kronecker coefficients. Other technics to prove the stability in case of plethysm coefficients can be found in papers of Manivel and Michałek [MM14] and Brion [Bri93], where they use vector bundles, or in papers of Carré and Thibon [CT92], with vertex operators technics.

\section{Plethysm coefficients: a combinatorial interpretation}

Any (finite-dimensional, complex, analytic) linear representation of $G L_{n}(\mathbb{C})$ can be completely described, up to isomorphism, by its character, which is a symmetric polynomial (see [Ful97, [FH91]). So, we can set the computations of plethysm in the framework of symmetric functions.

Recall that the ring of symmetric functions is a graded ring endowed with a scalar product (see [Ful97, Mac95, Sta99]). It admits several important linear basis as the Schur functions, $s_{\lambda}$; the monomial functions, $m_{\lambda}$, or the product of complete homogeneous functions, $h_{\lambda}=h_{\lambda_{1}} h_{\lambda_{2}} \ldots h_{\lambda_{k}}$. Observed that all the bases are indexed by partitions. With respect to the scalar product, the Schur functions are an orthonormal basis and the monomial functions and the complete homogeneous functions are dual bases.

The operation of plethysm in representation theory induces an operation $(f, g) \longrightarrow f[g]$ on the ring of symmetric functions, also called plethysm (see [Mac95]). Among the properties of plethysm, we remember that this operation is associative and linear in the first argument, but it is non-commutative and not bilinear.

In this framework, the plethysm coefficient $a_{\lambda, \mu}^{\nu}$ is the coefficient of $s_{\nu}$ in the expansion in the Schur basis of the plethysm of Schur functions $s_{\lambda}\left[s_{\mu}\right]$. Alternatively, due to the orthonormality of the Schur basis, this coefficient is extracted by means of a scalar product:

$$
a_{\lambda \mu}^{\nu}=\left\langle s_{\lambda}\left[s_{\mu}\right], s_{\nu}\right\rangle
$$

REMARK: It is important to recall that the plethysm coefficients have the following properties

$$
\left\langle s_{\lambda}\left[s_{\mu}\right], s_{\nu}\right\rangle=0 \quad \text { if }|\lambda| \cdot|\mu| \neq|\nu|
$$

Hence, the partitions we consider for coefficients $a_{\lambda, \mu}^{\nu}$ have the property that $|\lambda| \cdot|\mu|=|\nu|$.

The Jacobi-Trudi identity give us an expansion of $s_{\lambda}$ in terms of the complete homogeneous basis $\left\{h_{\gamma}\right\}$. 
We recall it here

Lemma 2.1 (Jacobi-Trudi identity, [Mac95] I. (3.4)) Let $\lambda$ be a partition with length at most $N$. Then

$$
s_{\lambda}=\operatorname{det}\left(h_{\lambda_{j}+i-j}\right)_{1 \leq i, j \leq N}
$$

with $h_{0}=1$ and $h_{r}=0$ if $r<0$, and $\lambda$ is completed with trailing zeros if necessary.

If we expand explicitly the determinant in this expression, we describe the Schur function as a sum over the permutations $\sigma$ in the symmetric group $\mathfrak{S}_{N}$ ([Mac95] I. (3.4'))

$$
s_{\lambda}=\sum_{\sigma \in \mathfrak{S}_{N}} \varepsilon(\sigma) h_{\lambda+\omega(\sigma)}
$$

where $\omega(\sigma)_{j}=\sigma(j)-j$ for all $j$ between 1 and $N$.

We now perform this Jacobi-Trudi expansion for $s_{\lambda}$ and $s_{\nu}$ in (1). We get the following alternating decomposition for the plethysm coefficients.

Lemma 2.2 Let $N$ and $N^{\prime}$ be positive integers. Let $\lambda, \mu$ and $\nu$ be partitions, such that $\lambda$ has length at most $N$ and $\nu$ has length at most $N^{\prime}$. Then

$$
a_{\lambda \mu}^{\nu}=\sum_{\sigma, \tau} \varepsilon(\sigma) \varepsilon(\tau)\left\langle h_{\lambda+\omega(\sigma)}\left[s_{\mu}\right], h_{\nu+\omega(\tau)}\right\rangle
$$

where the sum is carried over all permutations $\sigma \in \mathfrak{S}_{N}$ and $\tau \in \mathfrak{S}_{N^{\prime}}$.

We have expressed the plethysm coefficient $a_{\lambda, \mu}^{\nu}$ in terms of the h-plethysm coefficients, that are interesting for their own sake. For any partition $\mu$ and any finite sequences $\lambda$ and $\nu$ of integers we set:

$$
b_{\lambda \mu}^{\nu}=\left\langle h_{\lambda}\left[s_{\mu}\right], h_{\nu}\right\rangle .
$$

It turns out that these coefficients count the nonnegative solutions of systems of linear Diophantine equations whose constant terms depend linearly on the parts of $\lambda$ and $\nu$. In particular, they count integer points in polytopes with a nice description.

Before specifying a description of such polytopes, we should introduce some notation. For any partition $\mu$ and any positive integer $N$ let $t(\mu, N)$ be the set of semi-standard Young tableaux (see [Sta99] 7.10) of shape $\mu$ with entries between 1 and $N$. Let $\mathcal{P}_{\mu, N}=\left(\rho_{j}(T)\right)_{T, j}$ be the matrix whose rows are indexed by the tableaux $T \in t(\mu, N)$, whose columns are indexed by the integers $j$ between 1 and $N$, such that $\rho_{j}(T)$ is the number of occurrences of $j$ in $T$, ie. the row $T$ of $\mathcal{P}_{\mu, N}$ is the weight of the tableau $T$.

Proposition 2.3 Let $\lambda$ and $\nu$ finite sequences of positive integers and let $\mu$ be a partition. Let $\ell(\mu)$ be the length of $\mu$ and $N$ be at least the length of $\lambda, \mu$ and $\nu$.

The coefficient $b_{\lambda, \mu}^{\nu}$ is the cardinal of the set $Q(\lambda, \mu, \nu, N)$ of matrices $\mathcal{M}=\left(m_{i, T}\right)$ with nonnegative integer entries whose rows are indexed by the integers $i$ between 1 and $N$ and whose columns are indexed by the tableaux $T \in t(\mu, N)$ such that:

- Row sums CONDition of $\mathcal{M}$ : The sum of the entries in row $i$ of $\mathcal{M}$ is $\lambda_{i}$.

- Column sums COndition of $\mathcal{M P}_{\mu, N}$ : The sum of the entries in column $j$ of $\mathcal{M P}_{\mu, N}$ is $\nu_{j}$. 
The proof of Proposition 2.3 follows from working out with the expansion of the Schur polynomials into monomials and the definition of the plethysm as an evaluation.

We show an example how the result works: consider the partitions $\mu=(2), \lambda=\left(\lambda_{1}, \lambda_{2}\right)$ and $\nu=$ $\left(\nu_{1}, \nu_{2}, \nu_{3}\right)$ and take $N=3$. Firstly, we compute the set $t(\nu, N)$

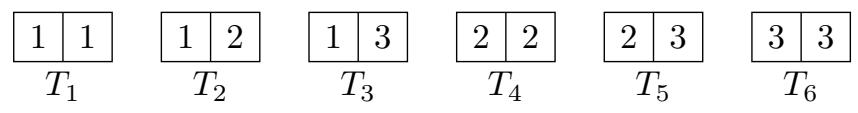

and the corresponding $\mathcal{P}_{\mu N}$ matrix

$$
\left(\begin{array}{lll}
2 & 0 & 0 \\
1 & 1 & 0 \\
1 & 0 & 1 \\
0 & 2 & 0 \\
0 & 1 & 1 \\
0 & 0 & 2
\end{array}\right)
$$

So, we are considering the matrices $\mathcal{M}$ of positive integer entries of the form

$$
\left(\begin{array}{cccccc}
m_{1 T_{1}} & m_{1 T_{2}} & m_{1 T_{3}} & m_{1 T_{4}} & m_{1 T_{5}} & m_{1 T_{6}} \\
m_{2 T_{1}} & m_{2 T_{2}} & m_{2 T_{3}} & m_{2 T_{4}} & m_{2 T_{5}} & m_{2 T_{6}} \\
m_{3 T_{1}} & m_{3 T_{2}} & m_{3 T_{3}} & m_{3 T_{4}} & m_{3 T_{5}} & m_{3 T_{6}}
\end{array}\right)
$$

that satisfy the following conditions

$$
\left\{\begin{array} { l } 
{ \sum _ { j } m _ { 1 T _ { j } } = \lambda _ { 1 } } \\
{ \sum _ { j } m _ { 2 T _ { j } } = \lambda _ { 2 } } \\
{ \sum _ { j } m _ { 3 T _ { j } } = 0 }
\end{array} \quad \left\{\begin{array}{l}
\sum_{i}\left(2 m_{i T_{1}}+m_{i T_{2}}+m_{i T_{3}}\right)=\nu_{1} \\
\sum_{i}\left(m_{i T_{2}}+2 m_{i T_{4}}+m_{i T_{5}}\right)=\nu_{2} \\
\sum_{i}\left(m_{i T_{3}}+m_{i T_{5}}+2 m_{i T_{6}}\right)=\nu_{3}
\end{array}\right.\right.
$$

\section{Proofs of stability properties}

Following the sketch of the proofs we give in the Introduction, once we have stablished which are the $h$-plethysm coefficients with the combinatorial interpretation, we have to proof the stability properties for them.

We show three stability properties. In each case, firstly we give the sketch of the proof for the property in case of h-plethysm coefficients $\left\{b_{\lambda(n), \mu(n)}^{\nu(n)}\right\}$ and then, as a corollary, we have the stability property for the plethysm coefficients $\left\{a_{\lambda(n), \mu(n)}^{\nu(n)}\right\}$.

\subsection{First stability property}

In this first case, we are going to consider the sequence of $h$-plethysm coefficients associated to the partitions

$$
\left\{\begin{array}{l}
\lambda(n)=\lambda+(n)=\left(\lambda_{1}+n, \lambda_{2}, \lambda_{3}, \ldots\right) \\
\mu(n)=\mu \\
\nu(n)=\nu+n \cdot \mu=\left(\nu_{1}+n \mu_{1}, \nu_{2}+n \mu_{2}, \nu_{3}+n \mu_{3}, \ldots\right)
\end{array}\right.
$$

This last operation can be seen as a sum of vectors, where we are adding zero parts in order to have the same length. 
Theorem 3.1 Let $\mu$ be a partition and $\lambda$ and $\nu$ be finite sequences of integers. Then, the sequence with general term $b_{\lambda+(n), \mu}^{\nu+n \cdot \mu}$ stabilizes.

Proof (Sketch): Let $N$ be an integer bigger than or equal to the lengths of $\lambda, \mu$ and $\nu$. After Proposition 2.3 ,

$$
b_{\lambda+(n), \mu}^{\nu+n \cdot \mu}=\operatorname{Card}(Q(\lambda+(n), \mu, \nu+n \cdot \mu, N)) .
$$

Set $E(n)=Q(\lambda+(n), \mu, \nu+n \cdot \mu, N)$.

Let $T_{1}$ be the tableau in $t(\mu, N)$ whose row number $i$ is filled with occurrences of $i$, for each $i$. Ie.

\begin{tabular}{|c|c|c|c|c|c|}
\hline 3 & 3 & 3 & & & \\
\hline 2 & 2 & 2 & 2 & & \\
\hline 1 & 1 & 1 & 1 & 1 & 1 \\
\hline
\end{tabular}

Consider the injection $\varphi_{n}: E(n) \hookrightarrow E(n+1)$ that maps any matrix $\mathcal{M} \in E(n)$ to the matrix $\mathcal{M}^{\prime}$ where the coefficient $m_{1, T_{1}}^{\prime}=m_{1, T_{1}}+1$ and all other coefficients are unchanged.

We contend that $\varphi_{n}$ is also surjective for $n$ big enough. Now, $\varphi_{n}$ is surjective if and only if for all $\mathcal{M}^{\prime} \in E(n+1)$, the entry $m_{1, T_{1}}^{\prime}$ is non-zero. So, in order to prove surjectivity, we will show that $m_{1, T_{1}}^{\prime}>0$.

Let $\mathcal{M}^{\prime} \in E(n+1)$. Observe that among all tableaux in $t(\mu, N)$, the tableau $T_{1}$ is the unique one with maximum weight for the dominance ordering ([Mac95] I.1). Denote $\|\alpha\|$ for $\sum_{j=1}^{N-1}(N-j) \cdot \alpha_{j}$ and $\rho(T)$ for row $T$ of $\mathcal{P}_{\mu, N}$. Then,

$$
\left\{\begin{array}{lll}
\|\rho(T)\| \leq\|\mu\|-1 & \text { if } & T \neq T_{1} \\
\left\|\rho\left(T_{1}\right)\right\|=\|\mu\| & \text { if } & T=T_{1}
\end{array}\right.
$$

Looking at the first column sums condition of $\mathcal{M P}_{\mu, N}$, a few more elementary calculations joint with the observation above, lead to the inequality

$$
m_{1, T_{1}}^{\prime} \geq\|\nu\|+\lambda_{1}-|\lambda| \cdot\|\mu\|+(n+1)
$$

that proves that $m_{1, T_{1}}^{\prime}>0$ as soon as $n \geq|\lambda| \cdot|| \mu||-\|\nu\|-\lambda_{1}$.

The relation between the coefficients $b_{\lambda \mu}^{\nu}$ and $a_{\lambda, \mu}^{\nu}$ that we have seen above shows that the stability in one case implies the stability in the other one. So, as a corollary we have the following result

Corollary 3.2 (Brion, [Bri93]) Let $\mu$ be a partition and $\lambda$ and $\nu$ be finite sequences of integers. Then, the sequence with general term $a_{\lambda+(n), \mu}^{\nu+n \cdot \mu}$ stabilizes.

\subsection{Second stability property}

The map we have defined in the theorem (3.1) can be also used in another stability properties, where $\lambda(n)=\lambda+(n)$ and $\mu(n)=\mu$. For example, we can look at the h-plethysm coefficients with $\nu(n)=$ $\nu+(|\mu| \cdot n)$. In that case, the coefficient will be null except when $\mu$ has just one part. 
Theorem 3.3 Let $\lambda, \mu$ and $\nu$ be three partitions. Consider the following $h$-plethysm coefficients

$$
b_{\lambda+(n), \mu}^{\nu+(|\mu| \cdot n)}=\left\langle h_{\lambda+(n)}\left[s_{\mu}\right], h_{\nu+(|\mu| \cdot n)}\right\rangle
$$

Then, we have that for $n$ big enough:

1. The sequence of coefficients stabilizes, when $\mu=(m)$.

2. The limit is zero, when $\ell(\mu)>1$.

Proof (Sketch): Let $N$ be an integer bigger than or equal to the lengths of $\lambda$ and $\nu$. By Proposition (2.3), we are considering the coefficients

$$
b_{\lambda+(n), \mu}^{\nu+(|\mu| \cdot n)}=\operatorname{Card}(Q(\lambda+(n), \mu, \nu+(|\mu| \cdot n), N))
$$

Set $E(n)=Q(\lambda+(n), \mu, \nu+(|\mu| \cdot n), N)$ as before.

1. We want to prove that there exists a bijection between $E(n)$ and $E(n+1)$, when $\mu=(m)$ and $n$ is big enough.

We define the map as in theorem (3.1)

$$
\begin{aligned}
& \varphi_{n}: E(n) \longrightarrow E(n+1) \\
& \mathcal{M} \longmapsto \mathcal{M}^{\prime}
\end{aligned}
$$

where $m_{1, T_{1}}^{\prime}=m_{1, T_{1}}+1$ and all other coefficients of $\mathcal{M}^{\prime}$ are unchanged.

Like $\varphi_{n}$ is well-defined and injective, we just need to prove that it is also surjective. As in the proof of Theorem 3.1 , we have to show that for all $\mathcal{M}^{\prime} \in E(n+1), m_{1, T_{1}}^{\prime}>0$.

In this case $\mu=(m)$, so our tableaux have only one row. Then, let $T_{1}$ be the tableaux in $t(\mu, N)$ which is filled just with ones. Then, this case is simplier and we do not need to consider the accumulated sums. It is enough to observe that $\rho\left(T_{1}\right)=(m, 0, \ldots, 0)$ will be the first row of $\mathcal{P}_{\mu, N}$. So, $\rho_{1}\left(T_{1}\right)=m$ and $\rho_{1}(T) \leq m-1$ for all $T \neq T_{1}$.

Using now the first column sums condition of $\mathcal{M}^{\prime} \mathcal{P}_{\mu, N}$ and a few more elementary operations, we see the following inequality

$$
\nu_{1}+n \cdot m \leq m_{1, T_{1}} \cdot m+(m-1)|\lambda|
$$

So, $m_{1, T_{1}}>0$ as soon as $n \geq \frac{|\nu|-\nu_{1}}{|\mu|}$.

2. We need to prove that $E(n)$ is empty for $n$ big enough.

The first column sums condition of $\mathcal{M P} \mathcal{P}_{\mu, N}$ says that

$$
\nu_{1}+|\mu| n=\sum_{i=1}^{N} \sum_{T \in t(\mu, N)} m_{i, T} \rho_{1}(T)
$$


In this case, we have the following bound for all tableaux

$$
\rho_{1}(T) \leq \mu_{1}
$$

because we cannot have ones in more boxes that the first row.

And we know also that

$$
\left(\sum_{i=1}^{N} \sum_{T \in t(\mu, N)} m_{i, T}\right)=\sum_{i=0}^{N} \lambda_{i}+n=|\lambda|+n
$$

So,

$$
\nu_{1}+|\mu| \cdot n \leq \mu_{1}(|\lambda|+n) \Longleftrightarrow n \leq \frac{\mu_{1} \cdot|\lambda|-\nu_{1}}{|\mu|-\mu_{1}}
$$

So, when $n>\frac{\mu_{1} \cdot|\lambda|-\nu_{1}}{|\mu|-\mu_{1}}$, there is no solution for our system of equations and inequations and $E(n)$ is an empty set.

Then, $b_{\lambda+(n), \mu}^{\nu+(|\mu| \cdot n)}=0$ as soon as $n>\frac{\mu_{1} \cdot|\lambda|-\nu_{1}}{|\mu|-\mu_{1}}$.

Corollary 3.4 (Carré and Thibon, [CT92]) The following plethysm coefficients

$$
a_{\lambda+(n), \mu}^{\nu+(|\mu| \cdot n)}=\left\langle s_{\lambda+(n)}\left[s_{\mu}\right], s_{\nu+(|\mu| \cdot n)}\right\rangle
$$

have the same behaviour when $n$ is big enough, ie.

1. The sequence of coefficients stabilizes when $\mu=(m)$.

2. The limit is zero when $\ell(\mu)>1$.

\subsection{Third stability property}

This last stability property is different in the following sense: in this case

$$
\left\{\begin{array}{l}
\lambda(n)=\lambda \\
\mu(n)=\mu+(n) \\
\nu(n)=\nu+(|\lambda| \cdot n)
\end{array}\right.
$$

So, what we are considering now is h-plethysm coefficients where the set of tableaux changes when $n$ grows.

Let see how is the proof in this case.

Theorem 3.5 Let $\lambda, \mu$ and $\nu$ be three partitions. The following $h$-plethysm coefficients

$$
b_{\lambda, \mu+(n)}^{\nu+(|\lambda| \cdot n)}=\left\langle h_{\lambda}\left[s_{\mu+(n)}\right], h_{\nu+(|\lambda| \cdot n)}\right\rangle
$$

stabilize. 
Proof (Sketch): Let $N$ be an integer bigger than or equal to the lengths of $\lambda$ and $\nu$. By Proposition (2.3), we are considering the coefficients

$$
b_{\lambda, \mu+(n)}^{\nu+(|\lambda| \cdot n)}=\operatorname{Card}(Q(\lambda, \mu+(n), \nu+(|\lambda| \cdot n), N))
$$

Set $E(n)=Q(\lambda, \mu+(n), \nu+(|\lambda| \cdot n), N)$ as before.

In this case, the set of tableaux changes in each step. So, first of all we define the following map

$$
\tau_{n}: t(\mu+(n), N) \longrightarrow t(\mu+(n+1), N)
$$

where $\tau_{n}(T)$ is obtained from $T$ by adding one box with a one in the first row and pushing the original

\begin{tabular}{|c|c|c|c|}
\hline 7 & & & \\
\hline 5 & & & \\
\hline 3 & 5 & & \\
\hline 2 & 3 & 3 & \\
\hline 1 & 2 & 2 & 2 \\
\hline
\end{tabular}
first row of $T$ to the right. Let see an example

$\tau_{n}$ is injective but it is not surjective. Then, we can separate $t(\mu+(n+1), N)$ into two sets: the set of tableaux with preimage, that we denote by $\bar{T}$, and the set of new tableaux, that we denote by $T^{\prime}$.

Now, in order to define $\varphi_{n}: E(n) \longrightarrow E(n+1)$, we are going to order the columns of the matrices. The first columns correspond to the tableaux with preimage and the next columns correspond to the new tableaux. So, the matrices are of the form $\mathcal{M}=\left(m_{i, \bar{T}} \mid m_{i, T^{\prime}}\right)$.

Then, we define $\varphi_{n}(\mathcal{M})=(\mathcal{M} \mid \overline{0})$. This means that we turn the matrix of $E(n), \mathcal{M}$, into a matrix of $E(n+1)$ by adding as many null columns as we need. In fact, we add in total the same number of columns as new tableaux there are in $t(\mu+(n+1), N)$.

$\varphi_{n}$ is well defined and it is clearly injective. Let see surjectivity:

Let $\mathcal{M}^{\prime} \in E(n+1)$ and consider the following reduction matrix $\left(\mathcal{M}_{\bar{T}}, \mathcal{M}_{T^{\prime}}\right)$, where $\mathcal{M}_{\bar{T}}=\sum_{i} m_{i, \bar{T}}$ and $\mathcal{M}_{T^{\prime}}=\sum_{i} m_{i, T^{\prime}}$. Let denote $S=\sum_{\bar{T}} \mathcal{M}_{\bar{T}}$ and $S^{\prime}=\sum_{T^{\prime}} \mathcal{M}_{T^{\prime}}$. Then, $S+S^{\prime}=|\lambda|$ and we need to show that $S^{\prime}=0$. Hence, all $m_{i, T^{\prime}}^{\prime}=0$ and $\mathcal{M}^{\prime}$ is of the form $(\mathcal{M} \mid \overline{0})$, with $\mathcal{M} \in E(n)$.

Looking at the first column sums condition of $\mathcal{M} \mathcal{P}_{\mu, N}$ condition, we can translate it into the following condition

$$
\nu_{1}+(n+1)|\lambda|=\sum_{\bar{T}} \mathcal{M}_{\bar{T}} \rho_{1}(\bar{T})+\sum_{T^{\prime}} \mathcal{M}_{T^{\prime}} \rho_{1}\left(T^{\prime}\right)
$$

Now, we can estimate the number of ones in each case

$$
\left\{\begin{array}{lll}
\rho_{1}(T) \leq \mu_{1}+n+1 & \text { if } & T=\bar{T} \\
\rho_{1}\left(T^{\prime}\right) \leq \mu_{2} & \text { if } & T=T^{\prime}
\end{array}\right.
$$

Using these estimations in (2) and doing some calculations, we get that

$$
\nu_{1}+(n+1) \cdot|\lambda| \leq|\lambda| \cdot\left(\mu_{1}+n+1\right)+\left(\mu_{2}-\mu_{1}-n-1\right) \cdot S^{\prime} \Longrightarrow S^{\prime} \leq \frac{\mu_{1} \cdot|\lambda|-\nu_{1}}{\mu_{1}+n+1-\mu_{2}}
$$


So, like $S^{\prime} \geq 0, S^{\prime}$ will be zero when $n>|\lambda| \cdot \mu_{1}-\nu_{1}-\mu_{1}-1+\mu_{2}$.

Once we have proved the result for the $b_{\lambda, \mu}^{\nu}$ coefficients, we obtain the corresponding property for the $a_{\lambda, \mu}^{\nu}$ coefficients.

Corollary 3.6 (Carré and Thibon, [CT92]) The following plethysm coefficients

$$
a_{\lambda, \mu+(n)}^{\nu+(|\lambda| \cdot n)}=\left\langle s_{\lambda}\left[s_{\mu+(n)}\right], s_{\nu+(|\lambda| \cdot n)}\right\rangle
$$

stabilizes.

\section{Conclusion}

This new approach provides a new proof of stability properties for plethysm coefficients using elementary tools of symmetric functions. Furthermore, it enhanced the importance of other constants (the $b_{\lambda, \mu}^{\nu}$ in this paper) that seem interesting by themselves. In particular, it should be possible to evaluate them efficiently, by means of Barvinok's algorithm. Could this lead to more efficient algorithms for computing the plethysm coefficients? Could this approach help us to prove more general stability properties?

\section{References}

[BO07] Cristina M. Ballantine and Rosa C. Orellana. A combinatorial interpretation for the coefficients in the Kronecker product $s_{(n-p, p)} * s_{\lambda}$. Sém. Lothar. Combin., 54A:Art. B54Af, 29 pp. (electronic), 2005/07.

[BOR11] Emmanuel Briand, Rosa Orellana, and Mercedes Rosas. The stability of the Kronecker product of Schur functions. J. Algebra, 331:11-27, 2011.

[Bri93] Michel Brion. Stable properties of plethysm: on two conjectures of Foulkes. Manuscripta Math., 80(4):347-371, 1993.

[CT92] Christophe Carré and Jean-Yves Thibon. Plethysm and vertex operators. Adv. in Appl. Math., 13(4):390-403, 1992.

[FH91] William Fulton and Joe Harris. Representation theory: A first course. Graduate Texts in Mathematics, Springer-Verlag, New York, 129, 1991.

[Fou50] H. O. Foulkes. Concomitants of the quintic and sextic up to degree four in the coefficients of the ground form. J. London Math. Soc., 25:205-209, 1950.

[Ful97] William Fulton. Young tableaux. London Mathematical Society Student Texts, Cambridge University Press, Cambridge, 35, 1997.

[Lit56] D. E. Littlewood. The Kronecker product of symmetric group representations. J. London Math. Soc., 31:89-93, 1956. 
[Mac95] I. G. Macdonald. Symmetric functions and Hall polynomials. Oxford Mathematical Monographs. The Clarendon Press, Oxford University Press, New York, second edition, 1995. With contributions by A. Zelevinsky, Oxford Science Publications.

[Man14] Laurent Manivel. On the asymptotics of Kronecker coefficients,. preprint, 2014. available at http://arxiv.org/pdf/1411.3498v1.pdf.

[MM14] Laurent Manivel and Mateusz Michałek. Effective constructions in plethysms and Weintraub's conjecture. Algebr. Represent. Theory, 17(2):433-443, 2014.

[Mur38] F. D. Murnaghan. The Analysis of the Kronecker Product of Irreducible Representations of the Symmetric Group. Amer. J. Math., 60(3):761-784, 1938.

[PP14] I. Pak and G. Panova. Bounds on the Kronecker coefficients. 2014.

[Sta99] Richard P. Stanley. Enumerative combinatorics. Vol. 2, volume 62 of Cambridge Studies in Advanced Mathematics. Cambridge University Press, Cambridge, 1999. With a foreword by Gian-Carlo Rota and appendix 1 by Sergey Fomin.

[Ste14] John R.1 Stembridge. Generalized stability of Kronecker coefficients,. preprint, 2014. available at http://www.math.lsa.umich.edu/ jrs/papers/kron.pdf.

[STW93] Thomas Scharf, Jean-Yves Thibon, and Brian G. Wybourne. Reduced notation, inner plethysms and the symmetric group. J. Phys. A, 26(24):7461-7478, 1993.

[Va199] Ernesto Vallejo. Stability of Kronecker products of irreducible characters of the symmetric group. Electron. J. Combin., 6:Research Paper 39, 7 pp. (electronic), 1999. 
